\title{
EXPONENTIATION OF REALS: EFFECTS OF BASE CHOICE
}

\author{
R. GUREVIC
}

\begin{abstract}
Let $r>1$ and $X_{r}$ be the minimal set of reals containing 1 and closed under $\exp _{r}: x \mapsto r^{x}$ and addition. The behavior of $X_{r}$ is studied. In particular among possible order types of $X_{r}$ there are $\omega, \omega^{\omega}, \omega+q, \omega+1+q$, where $q$ is the dense countable order without endpoints.
\end{abstract}

Let $r>0$ and $X_{r}$ be the minimal set of reals containing 1 and closed under $\exp _{r}$ : $x \mapsto r^{x}$ and addition. Obviously $X_{r}$ is dense in $\mathbf{R}_{+}$for $r<1$ and $X_{1}=\mathbf{N}$. I would like to describe the behavior of $X_{r}$ for $r>1$.

Preliminary remarks. (Proofs are provided in $\S 1$.) (i) $X_{r}=$ the set of values of constant $\left(1,+, \cdot, \exp _{r}\right)$-terms.

(ii) If $X$ is a well-ordered subset of $\mathbf{R}_{+}$closed under addition (or multiplication) then the order type of $X$ is $\omega^{\omega^{\alpha}}$ for some $\alpha$.

(iii) For any countable $\alpha$ if $X_{r}$ is well-ordered and its order type is $<\omega^{\omega^{\alpha}}$ then there exists a set $X \subset \mathbf{R}_{+}$containing $X_{r}$, closed under $+, \cdot, \exp _{r}$ and of order type $\omega^{\omega^{\alpha}}$.

Proposition 1. Let $a=\left(a^{a}+1\right)^{1 /\left(a^{a}+1\right)}$ and $b=s^{1 / s}$, where $s$ is the root of the equation $x^{1 / x}=(x+1)^{1 /(x+1)}$. (One can easily see that both equations have unique roots.) Then

(i) If $e^{1 / e}<r$ then $X_{r}$ has order type $\omega$.

(ii) If $a \leq r \leq e^{1 / e}$ then $X_{r}$ and $\bar{X}_{r}$ have order type $\omega^{\omega}$. $\varnothing$.

(iii) If $b<r<a$ then $X_{r}$ is not well-ordered and the Cantor derivative $\left(X_{r}\right)^{(\omega)}=$

(iv) $X_{b}$ is not well-ordered.

The Cantor derivative mentioned in (iii) is defined as follows: $Y^{(0)}=\bar{Y}, Y^{(\lambda+1)}$ $=$ the set of limit points of $Y^{(\lambda)}$, and $Y^{(\lambda)}=\bigcap\left\{Y^{(\mu)} \mid \mu<\lambda\right\}$ for limit $\lambda$.

Some numerical values:

$$
e^{1 / e}=1.4446678 \ldots, \quad a=1.4446575 \ldots, \quad b=1.4360782 \ldots
$$

CONJECTURE. If $1<r \leq b$ then $\bar{X}_{r}=\left\{\right.$ a sequence increasing to $\left.g_{r}\right\} \cup\left[g_{r}, \infty\right)$, where $g_{r}$ is the smallest root of the equation $r^{x}=x$.

Proposition 2. (i) If $1<r<b$ then $X_{r}$ contains a perfect subset i.e. a nonempty subset without isolated points.

(ii) The conjecture is valid for $1<r<1.4360782 \ldots$.

(iii) If $\ln \left(\ln \left(g_{b}\right)\right) / \ln \left(\ln \left(g_{b}+1\right)\right)$ is irrational then the conjecture is valid for $r=b$.

Received by the editors October 7, 1983 and, in revised form, August 29, 1984.

1980 Mathematics Subject Classification(1985 Revision). Primary 03E10, 11B05; Secondary 26A18.

Key words and phrases. Cantor derivative, perfect set, one-parameter iteration subgroup of the diffeomorphisms group. 
I am thankful to $\mathrm{H}$. Levitz for the advice to write down the results and to M. P. Katz for the proof of Lemma 8(i) in $§ 3$. My special thanks to the referee who requested a revision of the original paper: while doing that work I improved the results; the second revision concerned only expository difficulties.

Ends of proofs of claims and lemmata are marked with

1. Proofs of preliminary remarks. (i) I have to construct for any constant $\left(1,+, \cdot, \exp _{r}\right)$-term $t$ a constant $\left(1,+, \exp _{r}\right)$-term $u$ with the same value. Proceed by induction on the number of operations in $t$. Let $t=t_{1} t_{2}$, where $t_{1}, t_{2}$ are $\left(1,+, \exp _{r}\right)$-terms and $t_{1} \neq 1 \neq t_{2}$. If e.g. $t_{2}=u+v$ then we can apply the induction assumption to $t_{1} u, t_{1} v$ in $\left(t_{1} u\right)+\left(t_{1} v\right)$. If both $t_{1}, t_{2}$ are exponents, $t_{1}=r^{u}, t_{2}=r^{v}$, then the value of $t$ is the value of $\exp _{r}(u+v)$.

(ii) Let $\tau$ be the order type of $X$. It suffices to prove that if $\omega^{\lambda}<\tau$ then $\omega^{\lambda 2}<\tau$ for any $\lambda$, see [2, VII.7]. Let $X$ be closed under addition (the case of $X$ closed under multiplication is quite similar). Let $A$ be a bounded subset of $X$ with order type $\omega^{\lambda}$ and $x=\sup (A)$; for each $a \in A$ let $a^{+}$be its successor. Then for any $a \in A$ the order type of $\left\{a^{+}+b \mid b \in A \& a+x<a^{+}+b\right\}$ is $\omega^{\lambda}$, see [2, VII.6] (any nonzero remainder of $\omega^{\lambda}$ is $\omega^{\lambda}$ ). Thus $A+A$ has order type at least $\omega^{\lambda 2}$ and is bounded by $2 x$.

(iii) I will prove the following statement: for any set $A \subset \mathbf{R}_{+}$containing 1 and closed under $+, \cdot, \exp _{r}$ with order type $\omega^{\omega^{\alpha}}$, for any $p \in \mathbf{R}_{+}$, for any countable ordinal $\beta$ there exists a set $B \subset \mathbf{R}_{+}$closed under $+, \cdot, \exp _{r}$ with order type $\omega^{\omega^{\alpha+\beta}}$ and satisfying $B \supset A \& A \supset B \cap(0, p)$.

Proceed by induction on $\beta$. Consider first the limit case. Let $p>0$ and $\beta=$ $\lim \left\{\beta_{1}+\cdots+\beta_{n}\right\}_{n}$ with nonzero $\beta_{i}$ 's. Pick up $\left\{B_{i}\right\}_{i}$ as follows: let $B_{0}=A$ and $B_{i+1}$ be a set $\subset \mathbf{R}_{+}$such that $B_{i+1} \supset B_{i}, B_{i+1} \cap(0, p+i+1) \subset B_{i}, B_{i+1}$ is closed under $+, \cdot, \exp _{r}$ and its order type is $\omega^{\lambda_{i}}$, where $\lambda_{i}=\omega^{\alpha+\beta_{1}+\cdots+\beta_{i+1}}$. Put $B=\bigcup_{i} B_{i}$. Since any bounded subset of $B$ is contained in one of $B_{i}$ 's $B$ is wellordered and has order type at most $\omega^{\omega^{\alpha+\beta}}$, and since $B$ contains all $B_{i}$ 's its order type is at least $\omega^{\omega^{\alpha+\beta}}$.

Now turn to the case of $\beta=1$. Without loss of generality we can assume that $p>1$ and $r^{x}>x+1$ for $x \geq p$. Let $K$ be a subset of $(p, p+1)$ with order type $\omega^{\omega^{\alpha}}$ and $B$ be the minimal subset of $\mathbf{R}_{+}$closed under $+, \cdot, \exp _{r}$ and containing $A \cup K$. Since any bounded subset of $B$ is contained in a finite union of $\left(+, \cdot, \exp _{r}\right)$ combinations of $A, K$ the result follows from [3]:

Let $X, Y \subset \mathbf{R}_{+}$be well-ordered; then the order type of $X \cup Y$ is $\leq$ the Hessenberg natural sum of the order types of $X$ and $Y$, and the order types of $X+Y$ and $X \cdot Y$ are $\leq$ the Hessenberg natural product of the order types of $X$ and $Y$.

(This result is not very difficult, and you can prove it for your own pleasure.)

2. Proof of Proposition 1. For $r<e^{1 / e}$ let $g_{r}<h_{r}$ be the roots of the equation $x^{1 / x}=r$ (for $r=e^{1 / e}$ there is unique root $x=e$ and for $r>e^{1 / e}$ there are no roots). Clearly $g_{r}<e<h_{r}$, both $g_{r}$ and $h_{r}$ depend on $r \in\left(1, e^{1 / e}\right)$ differentiably and monotonically and tend to $e$ for $r \rightarrow e^{1 / e}$, and $r^{x}>x$ for $x \notin\left[g_{r}, h_{r}\right]$ and $r^{x}<x$ 
for $x \in\left(g_{r}, h_{r}\right)$. For convenience put also $g_{r}=h_{r}=e$ for $r=e^{1 / e}$. Denote $\exp _{r}^{0}$ : $x \mapsto x$ and $\exp _{r}^{n+1}(x)=\exp _{r}\left(\exp _{r}^{n}(x)\right)$. The sequence $\left\{\exp _{r}^{n}(1)\right\}_{n}$ increases and if bounded tends to $g_{r}$.

Claim 1. Let $T$ be an infinite set of constant $\left(1, g_{r},+, \exp _{r}\right)$-terms. If the set of values of terms $\in T$ is bounded then $T$ contains terms with arbitrarily high $\exp _{r}$-nests.

PROOF. If values of terms $\in T$ are bounded then there exists an $n$ such that each $t \in T$ consists of at most $n$ summands of the forms $1, g_{r}, r^{u}$. Thus the set of such summands is still infinite and the values of $u$ 's are bounded.

This provides (i) of the proposition: if $t$ contains an $\exp _{r}$-nest of height $n$ then $t \geq \exp _{r}^{n}(1)$, and $\left\{\exp _{r}^{m}(1)\right\}_{m}$ is unbounded for $r>e^{1 / e}$. On the other hand if $r \leq e^{1 / e}$ then $g_{r}$ is a limit point for $X_{r}$ and since $X_{r}$ is closed under addition all its finite number Cantor derivatives are nonempty.

Claim 2. Let $b<r \leq e^{1 / e}$ and $\left\{t_{n}\right\}_{n}$ be a sequence of constant $\left(1, g_{r},+, \exp _{r}\right)$ terms whose values are bounded. Then there exist finite sets $U$ of constant $\left(1,+, \exp _{r}\right)$-terms and $V$ of terms in variables and $1, g_{r},+, \exp _{r}$ such that each $t_{n}=$ $v\left(w_{1}, \ldots, w_{l}\right)$ for some $v \in V$, where each $w_{i}=\exp _{r}^{m}(u)$ for some $u \in U \cup\left\{g_{r}\right\}$ and $m$.

PROOF. Note first that there is a $k$ such that $\exp _{r}^{k}(1)+1>h_{r}$ (this follows from $\left.r>b: h_{b}=1+g_{b}=1+\lim \left\{\exp _{b}^{m}(1)\right\}_{m}\right)$. Let $K$ be the least such $k$. Note also that $\left\{\exp _{r}^{m}(x)\right\}_{m}$ is unbounded for $x>h_{r}$. Let the values of $t_{n}$ 's be $<M$ and $J$ be the least $j$ such that $\exp _{r}^{j}\left(\exp _{r}^{K}(1)+1\right)>M$.

Let $U=\{1\} \cup\left\{u \mid u\right.$ is a sum and appears as a subterm of some $t_{n}$ in the scope of at least $J \exp _{r}$ 's $\}$. I will show that $U$ is finite. Note that the values of terms $\in U$ are bounded by $\exp _{r}^{K}(1)+1$. If $U$ was infinite then Claim 1 would tell us that elements of $U$ contain arbitrarily high $\exp _{r}$-nests which is impossible in view of the above bound and the fact that the nontrivial elements of $U$ are sums. If a term $u \in U$ contained $g_{r}$ we would have $u=q+s \geq g_{r}+1>\exp _{r}^{K}(1)+1$; thus elements of $U$ are $\left(1,+, \exp _{r}\right)$-terms.

To obtain $V$ imagine $t_{n}$ 's typewritten on sheets of paper so that each $\exp _{r}(w)$ is displayed as $r^{w}$ and substitute by variables the parts of $t_{n}$ 's which are above $J$. One can express this rigorously as follows. For a subterm $u$ of $w$ let height $(u, w)=$ the number of $\exp _{r}$ 's in $w$ affecting $u$; and let height $(w)=1+\max \{\operatorname{height}(u, w) \mid$ $u$ is a subterm of $w\}$. Present each $t_{n}$ with height $J$ in the form $v\left(w_{1}, \ldots, w_{l}\right)$, where $v$ is a term in variables and $1, g_{r},+, \exp _{r}, w_{i}$ 's are constant $\left(1, g_{r},+, \exp _{r}\right)$ terms, and height $(v)=J+1$, height $\left(\operatorname{variable}_{i}, v\right)=\operatorname{height}\left(w_{i}, t_{n}\right)=J$ for each $i$. Let $V$ be the set of such $v$ 's. To show that $V$ is finite note first that the values of $\{v(1, \ldots, 1) \mid v \in V\}$ are bounded $(<M)$; now reasoning similar to that in the proof of Claim 1 provides the result.

COROLlaRY 3. If $b<r \leq e^{1 / e}$ then $\left(X_{r}\right)^{(m)}=\{$ the values of constant $\left(1, g_{r},+, \exp _{r}\right)$-terms with $\geq m$ occurrences of $\left.g_{r}\right\}$ for any $m$, and $\left(X_{r}\right)^{(\omega)}=\varnothing$.

PROOF. Proceed by induction on $n$. Let $x \in\left(X_{r}\right)^{(n+1)}$,

$$
x=\lim \left\{\text { value of } t_{m}\right\}_{m},
$$

each $t_{m}$ being a constant $\left(1, g_{r},+, \exp _{r}\right)$-term with $\geq n$ occurrences of $g_{r}$ and 
the values of $t_{m}$ 's distinct. In view of Claim 2 we can assume that each $t_{m}=$ $u\left(v_{m}, \ldots, w_{m}\right)$ for a term $u$ in variables and $1, g_{r},+, \exp _{r}$ with $\geq n$ occurrences of $g_{r}$ and $v_{m}=\exp _{r}^{j(m)}(v), \ldots, w_{m}=\exp _{r}^{k(m)}(w)$ for some constant $\left(1,+, \exp _{r}\right)$ terms $v, \ldots, w$ with increasing $j(), \ldots, k()$. Thus $x=$ the value of $u\left(g_{r}, \ldots, g_{r}\right)$. Conversely the value of $t\left(g_{r}\right)$ is $\lim \left\{\text { the value of } t\left(\exp _{r}^{m}(1)\right)\right\}_{m}$. Besides, Claim 2 shows that if $\left\{t_{m}\right\}_{m}$ is a bounded sequence of constant $\left(1, g_{r},+, \exp _{r}\right)$-terms then \{the number of occurrences of $g_{r}$ in $\left.t_{m}\right\}_{m}$ is bounded, whence $\left(X_{r}\right)^{(\omega)}=\varnothing$.

COROLLARY 4. If $b<r \leq e^{1 / e}$ and $X_{r}$ is well-ordered then its and $\bar{X}_{r}$ 's order types are both $\omega^{\omega}$.

PROOF. Immediately from the preliminary remark (ii) and the above corollary.

Clearly $r^{r^{r}+1}<r^{r}+1$ for $r<a$ whence $\left\{\exp _{r}^{m}\left(r^{r}+1\right)\right\}_{m}$ decreases for $r<a$ which gives (iii), (iv) of the proposition. I will show that $X_{r}$ is well-ordered for $r \geq a$.

Claim 5. If $X_{r}$ is not well-ordered then there exists a sequence $\left\{t_{m}\right\}_{m}$ of $\left(1,+, \exp _{r}\right)$-terms such that $t_{m}>\exp _{r}\left(t_{m+1}\right)$ for each $m$.

PROOF. Let $\left\{s_{m}\right\}_{m}$ be a decreasing sequence of $\left(1,+, \exp _{r}\right)$-terms. Put $t_{0}=s_{0}$. Since the values of $\left\{s_{m}\right\}_{m}$ are bounded the number of summands in $\left.s_{m}\right\}_{m}$ is also bounded. Consequently (turning if necessary to a subsequence) we may assume that there exists a sequence $\left\{s_{m}^{\prime}\right\}_{m \geq 1}$ of $\left(1,+, \exp _{r}\right)$-terms such that each $\exp _{r}\left(s_{m}^{\prime}\right)$ is a summand of $s_{m}$ and the values of $\left\{s_{m}^{\prime}\right\}_{m \geq 1}$ decrease. Of course the value of $t_{0}$ is greater than those of $\left\{\exp _{r}\left(s_{m}^{\prime}\right)\right\}_{m \geq 1}$. Apply the same procedure to $\left\{s_{m}^{\prime}\right\}_{m \geq 1}$ and proceed $\omega$ times.

Claim 6. Let $b<r \leq e^{1 / e}$. If $X_{r}$ is not well-ordered then there exists a $\left(1,+, \exp _{r}\right)$-term $t$ such that $r^{t}<t$.

PROOF. Let $\left\{t_{m}\right\}_{m}$ be a sequence of $\left(1,+, \exp _{r}\right)$-terms such that $t_{m}>$ $\exp _{r}\left(t_{m+1}\right)$ for each $m$ (see Claim 5). If $t_{m}>h_{r}$ then $\&_{k} t_{m+k} \leq x_{k}$, where $x_{k+1}=\log _{r}\left(x_{k}\right), x_{0}=$ the value of $t_{m} ;$ and $\left\{x_{k}\right\}_{k}$ converges to $h_{r}$. Thus the values of $t_{m}$ 's are bounded. Since for $k>0$ inequality $\exp _{r}^{k}(x)<x$ holds iff $x \in\left(g_{r}, h_{r}\right)$ it will be enough to find a $t$ with $t>\exp _{r}^{k}(t)$ for some $k>0$ : then $g_{r}<t<h_{r}$ and $r^{t}<t$.

If for some $m<n$ we have $t_{m}=t_{n}$ then we have $t_{m}>\exp _{r}^{n-m}\left(t_{m}\right)$. Suppose that all $t_{m}$ 's are distinct. Then by Claim 2 there exist an increasing sequence $s$ of positive integers, a term $v$ in variables and $1,+, \exp _{r}$, constant $\left(1,+, \exp _{r}\right)$-terms $u_{1}, \ldots, u_{l}$ and increasing sequences $k_{1}, \ldots, k_{l}$ of positive integers such that for each $m$ we have $t_{s(m)}=v\left(\exp _{r}^{k_{1}(m)}\left(u_{1}\right), \ldots, \exp _{r}^{k_{1}(m)}\left(u_{l}\right)\right)$. If $\exp _{r}^{k_{i}(1)}\left(u_{i}\right)>\exp _{r}^{k_{i}(2)}\left(u_{i}\right)$ for at least one $i$ then we have $u_{i}>\exp _{r}^{k_{i}(2)-k_{i}(1)}\left(u_{i}\right)$. Otherwise we have

$$
\begin{aligned}
t_{s(1)} & >\exp _{r}^{s(2)-s(1)}\left(t_{s(2)}\right) \\
& =\exp _{r}^{s(2)-s(1)}\left(v\left(\exp _{r}^{k_{1}(2)}\left(u_{1}\right), \ldots, \exp _{r}^{k_{1}(2)}\left(u_{l}\right)\right)\right) \\
& \geq \exp _{r}^{s(2)-s(1)}\left(v\left(\exp _{r}^{k_{1}(1)}\left(u_{1}\right), \ldots, \exp _{r}^{k_{1}(1)}\left(u_{l}\right)\right)\right) \\
& =\exp _{r}^{s(2)-s(1)}\left(t_{s(1)}\right) .
\end{aligned}
$$

Claim 7. If $a \leq r \leq e^{1 / e}$ then $X_{r}$ is well-ordered.

PROOF. Suppose the contrary; let $t$ be a $\left(1,+, \exp _{r}\right)$-term such that $r^{t}<t$. Without loss of generality we can assume that $t$ is not an exponent (if $t=r^{u}$ then 
$r^{r^{u}}<r^{u}$ implies $r^{u}<u$ ). Consequently $t$ is a sum. If $t$ has three or more summands then its value is $\geq 3>h_{a} \geq h_{r}$ contradicting the choice of $t$ (for $x>h_{r}$ implies $\left.r^{x}>x\right)$. Thus $t$ has two summands. If both summands of $t$ are $\neq 1$ then they are $\geq r$ whence $t \geq 2 r \geq 2.889 \ldots>h_{a} \geq h_{r}$ (again impossible). On the other hand $2<g_{a} \leq g_{r}$ whereas $r^{x}>x$ for $x<g_{r}$. Thus $t=1+r^{u}$. If $u$ is a sum then $t \geq$ $1+r^{2}>1+2>h_{a} \geq h_{r}$. If $u=1$ then the value of $t$ is $1+r \leq 2.444 \ldots<g_{a} \leq g_{r}$. Thus $u=r^{v}$. If $v$ is a sum then $t \geq 1+r^{r^{2}}>1+r^{2}>3>h_{a} \geq h_{r}$. If $v=r^{w}$ then $t \geq 1+r^{r^{r}} \geq 1+a^{a^{a}}=2.8699218 \ldots>h_{a} \geq h_{r}$. Thus $v=1$ and $t=1+r^{r}$. However $\exp _{r}\left(1+r^{r}\right) \geq 1+r^{r}$ for $r \geq a$ by definition of $a$.

\section{Proof of Proposition 2.}

LEMMA 1. Let $f \in C^{1}([p, q]), f(p)=p, f(q) \geq p+1$ and $0<f^{\prime}(x)<1$ for $x \in$ $(p, q)$. Let $Y$ be the subset of $[p, q)$ minimal with respect to the properties: $p \in Y$, $x \in Y \& x+1<q \Rightarrow x+1 \in Y, x \in Y \Rightarrow f(x) \in Y$. Then $\bar{Y}=[p, q]$.

ProOF. Suppose the contrary. Any open interval $\subset(p, q)-\bar{Y}$ will be called a hole. Since $\bar{Y}$ is closed under $+1, f$ we have $\bar{Y} \supset[p, q] \cap\left(\bigcup_{m=0}^{\infty} m+f(\bar{Y})\right)$ whence the length of any hole is bounded by that of a hole $\subset(p, p+1) \subset(p, f(q))$. On the other hand $f$ maps $(p, q)$ onto $(p, f(q))$ monotonically with derivative $<1$, so any hole $\subset(p, f(q))$ is the $f$-image of a larger one. Thus we obtain a contradiction: let $H$ be a hole with maximal length, let $H^{\prime}$ be a hole $\subset(p, p+1)$ with length $\left(H^{\prime}\right) \geq \operatorname{length}(H)$, let $H^{\prime \prime}=f^{-1}\left(H^{\prime}\right)$, then length $\left(H^{\prime \prime}\right)>\operatorname{length}(H)$.

COROLLARY 2. Let $c=s^{1 / s}$, where $s$ is the root of the equation $x+1=x / \ln (x)$. Then $\left[g_{r}, \infty\right) \subset \bar{X}_{r}$ for $1<r<c(c=1.3998168 \ldots)$.

Proof. Put $d=-\ln (\ln (r)) / \ln (r)$. Then $\left(r^{x}\right)^{\prime}=r^{x} \cdot \ln (r)<1$ for $x<d$ and $g_{r}+1 \leq r^{d}<d<h_{r}$ for $r<c$. So the result follows from Lemma 1 applied to $\exp _{r} \in C^{1}\left(\left[g_{r}, d\right]\right)$.

Now let us turn to $r \in(c, b)$. A real $x \in\left[g_{r}+1, \log _{r}\left(g_{r}+1\right)\right)$ will be called tame if it is the value of a constant $\left(1, g_{r},+, \exp _{r}\right)$-term. Clearly $\{$ tame reals $\} \subset \bar{X}_{r}$.

Claim 3. If the set of tame reals is dense in $\left(g_{r}+1, \log _{r}\left(g_{r}+1\right)\right)$ then $X_{r}$ is dense in $\left(g_{r}, \infty\right)$.

PROOF. If tame reals are dense in $\left(g_{r}+1, \log _{r}\left(g_{r}+1\right)\right)$ then $X_{r}$ is also dense there and consequently $X_{r}$ is dense in $\bigcup_{k=0}^{\infty} \exp _{r}^{k}\left(\left[g_{r}+1, \log _{r}\left(g_{r}+1\right)\right)\right.$ which is just $\left(g_{r}, \log _{r}\left(g_{r}+1\right)\right)$ since $g_{r}=\lim \left\{\exp _{r}^{k}\left(g_{r}+1\right)\right\}_{k}$ for $r<b$. Thus $X_{r}$ is dense in $\left[g_{r}, g_{r}+1\right) \subset\left[g_{r}, \log _{r}\left(g_{r}+1\right)\right)$ and so $X_{r}$ is dense in $\left[g_{r}, \infty\right)$.

So, $I$ intend to prove that tame reals are dense in $\left[g_{r}+1, \log _{r}\left(g_{r}+1\right)\right)$ for $c \leq r<1.4360782 \ldots$ and I conjecture that this will hold for any $r \in(c, b)$. For any sequence $m=\left\{m_{k}\right\}_{k \geq 1}$ of positive integers let $\left\{m \mathcal{L}_{k}\right\}_{k}$ be the following sequence of one-variable $\left(1,+, \exp _{r}\right)$-terms: $m \mathcal{L}_{0}(x)=x, m \mathcal{L}_{k}(x)=m \mathcal{L}_{k-1}\left(\exp _{r}^{m_{k}}(x)+1\right)$ for $k \geq 1$.

Claim 4. For any $x \in\left(g_{r}+1, \log _{r}\left(g_{r}+1\right)\right)$ there exist unique $l \in \mathbf{N}$ and $y \in\left[g_{r}+\right.$ $\left.1, \log _{r}\left(g_{r}+1\right)\right)$ such that $x=\exp _{r}^{l}(y)+1$. For any nontame $x \in\left[g_{r}+1, \log _{r}\left(g_{r}+1\right)\right)$ there exist unique sequences $\left\{m_{k}\right\}_{k \geq 1}$ of positive integers and $\left\{y_{k}\right\}_{k \geq 0}$ of reals $\in\left(g_{r}+1, \log _{r}\left(g_{r}+1\right)\right)$ such that $x=m \mathcal{L}_{k}\left(y_{k}\right)$ for each $k$.

PROOF. The first part of the claim is an immediate consequence of the facts that $\log _{r}\left(g_{r}+1\right)-\left(g_{r}+1\right)<1$ for $r \geq c$ (existence) and that $\left(g_{r}, \log _{r}\left(g_{r}+1\right)\right)$ is the disjoint union of $\left\{\exp _{r}^{l}\left[g_{r}+1, \log _{r}\left(g_{r}+1\right)\right)\right\}_{l=0}^{\infty}$ for $r<b$ (uniqueness). For the 
second part of the claim proceed by induction: if we have already $y_{k}$ (put $y_{0}=x$ ) then $y_{k} \neq g_{r}+1$ (since $x$ is not tame) and by the first part of the claim we get $m_{k+1}$ and $y_{k+1}$.

Claim 5. Tame reals are dense in $\left(g_{r}+1, \log _{r}\left(g_{r}+1\right)\right)$ for $c \leq r<1.4360782 \ldots$ (this completes (ii) of Proposition 2).

PROOF. Let $x \in\left[g_{r}+1, \log _{r}\left(g_{r}+1\right)\right)$ be nontame and $\left\{m_{k}\right\}_{k},\left\{y_{k}\right\}_{k}$ be as in Claim 4. We have $x=m \mathcal{L}_{k}\left(y_{k}\right)$ for each $k$ and I am going to prove that $x=\lim \left\{m \mathcal{L}_{k}\left(g_{r}+1\right)\right\}_{k}$. Note that functions $\left\{m \mathcal{L}_{k}\right\}_{k}$ and their derivatives are positive and increasing (except the trivial case $k=0$ ). Since

$$
\begin{aligned}
m \mathcal{L}_{k}\left(y_{k}\right)-m \mathcal{L}_{k}\left(g_{r}+1\right)< & \left(y_{k}-g_{r}-1\right) \cdot\left(\left.\frac{d}{d t} m \mathcal{L}_{k}(t)\right|_{t=y_{k}}\right) \\
& <\left(\log _{r}\left(g_{r}+1\right)-g_{r}-1\right) \cdot\left(\left.\frac{d}{d t} m \mathcal{L}_{k}(t)\right|_{t=y_{k}}\right)
\end{aligned}
$$

and $\log _{r}\left(g_{r}+1\right)-g_{r}-1<1$ for $r \geq c$, it suffices to show that $\left.\left(m \mathcal{L}_{k}(t)\right)^{\prime}\right|_{t=y_{k}}$ tends to 0 while $k \rightarrow \infty$. Since

$$
\begin{aligned}
\left.\left(m \mathcal{L}_{k}(t)\right)^{\prime}\right|_{t=y_{k}} & =\left.\left(m \mathcal{L}_{k-1}\left(\exp _{r}^{m_{k}}(t)+1\right)\right)^{\prime}\right|_{t=y_{k}} \\
& =\left.\left.\left(m \mathcal{L}_{k-1}(t)\right)^{\prime}\right|_{t=y_{k-1}} \cdot\left(\exp _{r}^{m_{k}}(t)+1\right)^{\prime}\right|_{t=y_{k}}
\end{aligned}
$$

and

$$
\left.\left(\exp _{r}^{m_{k}}(t)+1\right)^{\prime}\right|_{t=y_{k}}<\left.\left(\exp _{r}^{m_{k}}(t)+1\right)^{\prime}\right|_{t=\log _{r}\left(g_{r}+1\right)}
$$

it will be enough to prove that $\sup \left\{\left.\left(\exp _{r}^{m_{k}}(t)\right)^{\prime}\right|_{t=\log _{r}\left(g_{r}+1\right)}\right\}_{k}<1$. Well,

$$
\left(\exp _{r}^{n}(t)\right)^{\prime}=\prod_{j=1}^{n}\left(\ln (r) \cdot \exp _{r}^{j}(t)\right)
$$

and its factors $\left\{\ln (r) \cdot \exp _{r}^{j}(t)\right\}_{j}$ decrease with increasing $j$ for any fixed $t \in\left(g_{r}, h_{r}\right)$. Consequently if $\left(\exp _{r}^{n}(t)\right)^{\prime}<1$ at some $t \in\left(g_{r}, h_{r}\right)$ then $\left(\exp _{r}^{m}(t)\right)^{\prime}<1$ at the same $t$ whenever $m \geq n$. Let $J=J(r)$ be the minimal positive integer with the property $\exp _{r}^{J}\left(g_{r}+1\right)+1<\log _{r}\left(g_{r}+1\right)$. Then $m_{k} \geq J$ for all $k$ and the claim will go through whenever $\left.\left(\exp _{r}^{J}(t)\right)^{\prime}\right|_{t=\log _{r}\left(g_{r}+1\right)}<1$. I checked this by machine computations (with long reals) for $c \leq r<1.4360782 \ldots$ (corresponding to $J \leq 150$ ).

REMARK. Actually I conjecture that the inequality $\left.\left(\exp _{r}^{J(r)}(t)\right)^{\prime}\right|_{t=\log _{r}\left(g_{r}+1\right)}<1$ (see above) holds for all $r \in(c, b)$. Moreover, it is possible to extend $\left\{\exp _{r}^{k}\right\}_{k}$ to a one-parameter iteration subgroup of the diffeomorphisms group of $\left(g_{r}, h_{r}\right)$, and I conjecture that

$$
\frac{d}{d r}\left(\frac{d}{d t} \exp _{r}^{j(r)}(t) \text { at } t=\log _{r}\left(g_{r}+1\right)\right)<0 \quad \text { for } c \leq r<b,
$$

where $j(r)$ is a differentiable function of $r$ determined by equation $\exp _{r}^{j}\left(g_{r}+1\right)+1=$ $\log _{r}\left(g_{r}+1\right)$.

Claim 6. There exists $K \in \mathbf{N}$ such that $\left.\left(\exp _{r}^{M}(t)\right)^{\prime}\right|_{t=\log _{r}\left(g_{r}+1\right)}<1$ for $M \geq K$.

PROOF. $\left(\exp _{r}^{M}(t)\right)^{\prime}=\prod_{j=1}^{M}\left(\ln (r) \cdot \exp _{r}^{j}(t)\right)$ and $\lim \left\{\ln (r) \cdot \exp _{r}^{j-1}\left(g_{r}+1\right)\right\}_{j}=$ $\ln (r) \cdot g_{r}=\ln \left(g_{r}\right)<1$. Consequently $\ln (r) \cdot \exp _{r}^{j-1}\left(g_{r}+1\right)<$ const $<1$ for sufficiently large $j$ 's and $\left\{\left.(d / d t) \exp _{r}^{M}(t)\right|_{t=\log _{r}\left(g_{r}+1\right)}\right\}_{M}$ tends to 0 for $M \rightarrow \infty$.

Claim 7. For any $r \in(c, b)$ there is an embedding of the Cantor binary set $\{0,1\}^{\mathbf{N}}$ into $\bar{X}_{r}$. (This provides (i) of the proposition.) 
PROOF. Take $K \in \mathbf{N}$ so large that $\left.\left(\exp _{r}^{K}(t)\right)^{\prime}\right|_{t=\log _{r}\left(g_{r}+1\right)}<1$ (see Claim 6) and $\exp _{r}^{K-1}\left(g_{r}+1\right)+1<\log _{r}\left(g_{r}+1\right)$. The latter implies that for any sequence $\left\{m_{k}\right\}_{k}$ of positive integers satisfying $\&_{k} m_{k} \geq K$ we have

$$
\&_{k} m \mathcal{L}_{k}\left[g_{r}+1, \log _{r}\left(g_{r}+1\right)\right) \Subset\left[g_{r}+1, \log _{r}\left(g_{r}+1\right)\right) .
$$

Evidently $\left\{m \mathcal{L}_{k}\left(g_{r}+1\right)\right\}_{k}$ is increasing and in view of the above inclusion is also bounded. For any 0 -1-sequence $1=\left\{l_{k}\right\}_{k=1}^{\infty}$ let $f(1)=\lim \left\{m \mathcal{L}_{k}\left(g_{r}+1\right)\right\}_{k}$, where $m_{k}=K+l_{k}$. It remains to check that $f$ is continuous and injective.

Injectivity. Recall that $f\left(\{0,1\}^{\mathbf{N}}\right) \subset\left(g_{r}+1, \log _{r}\left(g_{r}+1\right)\right)$. Let $\mathbf{k}, \mathbf{l} \in\{0,1\}^{\mathbf{N}}$ coincide in the first $n-1$ entries and $k_{n}=0, l_{n}=1$. Let $\mathbf{k}_{q}, \mathbf{l}_{q}$ denote the $(q+1)$ tails of $\mathbf{k}, \mathbf{l}$ respectively i.e. $\left(\mathbf{k}_{q}\right)_{p}=k_{q+p},\left(\mathbf{l}_{q}\right)_{p}=l_{q+p}$ for $q \in \mathbf{N}$. We have $f\left(\mathbf{k}_{n}\right)$, $f\left(\mathbf{l}_{n}\right) \in\left(g_{r}+1, \log _{r}\left(g_{r}+1\right)\right)$ and

$$
\left\{\begin{array}{l}
f\left(\mathbf{k}_{n-1}\right) \in 1+\exp _{r}^{K}\left(g_{r}+1, \log _{r}\left(g_{r}+1\right)\right), \\
f\left(\mathbf{l}_{n-1}\right) \in 1+\exp _{r}^{K+1}\left(g_{r}+1, \log _{r}\left(g_{r}+1\right)\right)
\end{array}\right.
$$

with disjoint right-hand side sets. Since $p \mathcal{L}_{n-1}$, where $p$ is $K+($ the common beginning of $\mathbf{k}, \mathbf{l})$, is a strictly increasing function and $f(\mathbf{k})=p \mathcal{L}_{n-1}\left(f\left(\mathbf{k}_{n-1}\right)\right)$, $f(\mathbf{l})=p \mathcal{L}_{n-1}\left(f\left(\mathbf{l}_{n-1}\right)\right)$ we have $f(\mathbf{k}) \neq f(\mathbf{l})$.

Continuity. Denote $\left.\left(\exp _{r}^{K}(t)\right)^{\prime}\right|_{t=\log _{r}\left(g_{r}+1\right)}=w, w<1$. Then

$$
\sup \left\{(d / d t) m \mathcal{L}_{k}(t) \mid t \in\left(g_{r}+1, \log _{r}\left(g_{r}+1\right)\right)\right\} \leq w^{k}
$$

whenever $\&_{k} m_{k} \geq K$. Consequently if $\mathbf{k}, \mathbf{l} \in\{0,1\}^{\mathbf{N}}$ coincide in the first $n$ entries then $|f(\mathbf{k})-f(\mathbf{l})|<\left(\log _{r}\left(g_{r}+1\right)-g_{r}-1\right) \cdot w^{n}$.

LEMMA 8. Let $f \in C^{2}(\mathbf{R}), f(0)=0, f^{\prime}(0) \in \mathbf{R}_{+}-\{1\}$. For any $x_{0} \in \mathbf{R}_{+}$set $x_{k}=f\left(x_{k-1}\right), k \geq 1$. Then $\forall s>0: \exists q>0$ : for any $n$,

(i) $x_{0}(1-s)<x_{n}\left(f^{\prime}(0)\right)^{-n}<x_{0}(1+s)$,

(ii) $\left(x_{0}-y_{0}\right)(1-s)<\left(x_{n}-y_{n}\right)\left(f^{\prime}(0)\right)^{-n}<\left(x_{0}-y_{0}\right)(1+s)$,

whenever $0<y_{0}<x_{0}<q$ for $f^{\prime}(0)<1$ or $0<y_{n}<x_{n}<q$ for $f^{\prime}(0)>1$.

PROOF. It suffices to consider only $f^{\prime}(0)<1$. We have $x_{k}=f^{\prime}(0) x_{k-1}+u_{k} x_{k-1}^{2}$ with $u_{k}=f^{\prime \prime}\left(a\right.$ real $\left.\in\left(0, x_{k-1}\right)\right) / 2$ and $\left\{u_{k}\right\}_{k}$ is bounded. So

$$
x_{k} / x_{k-1}=f^{\prime}(0) \cdot\left(1+u_{k} x_{k-1} / f^{\prime}(0)\right)
$$

and

$$
x_{n} / x_{0}=\left(f^{\prime}(0)\right)^{n} \cdot \prod_{k=1}^{n}\left(1+u_{k} x_{k-1} / f^{\prime}(0)\right) .
$$

We also have $x_{k}-y_{k}=\left(x_{k-1}-y_{k-1}\right) v_{k}$ with $v_{k}=f^{\prime}\left(a\right.$ real $\left.\in\left(y_{k-1}, x_{k-1}\right)\right)=$ $f^{\prime}(0)+w_{k} x_{k-1}$ with $w_{k}=(a$ real $\in(0,1)) \cdot f^{\prime \prime}\left(a\right.$ real $\left.\in\left(0, x_{k-1}\right)\right)$ and $\left\{w_{k}\right\}_{k}$ is bounded. So

$$
\left(x_{n}-y_{n}\right) /\left(x_{0}-y_{0}\right)=\left(f^{\prime}(0)\right)^{n} \cdot \prod_{k=1}^{n}\left(1+w_{k} x_{k-1} / f^{\prime}(0)\right) .
$$

And since for sufficiently small $x_{0}$ we have $\&_{n} x_{n}<x_{0} \cdot t^{n}$ for any $t>f^{\prime}(0)$, both products $\prod_{k}\left(1+u_{k} x_{k-1} / f^{\prime}(0)\right)$ and $\prod_{k}\left(1+w_{k} x_{k-1} / f^{\prime}(0)\right)$ can be made arbitrarily close to 1 by appropriate choice of $q$. 
From now on assume that $\ln \left(\ln \left(g_{b}\right)\right) / \ln \left(\ln \left(h_{b}\right)\right)$ is irrational. Consider the set

$$
W=\left\{\exp _{b}^{m}\left(\exp _{b}^{n}(1)+1\right) \mid m, n \in \mathbf{N} \& \exp _{b}^{n}(1)+1>g_{b}\right\}
$$

Clearly $W \subset\left(g_{b}, h_{b}\right)$. I will prove that $W$ is dense in $\left(g_{b}, h_{b}\right)$.

Claim 9. For any $s>0$ there exists a sequence $\left\{w_{k}\right\}_{k \in \mathbf{N}} \subset W$ such that $w_{k}<w_{k+1} \&\left(h_{b}-w_{b}\right) /\left(h_{b}-w_{k+1}\right)<(1+s)^{5} /(1-s)^{5}$ for all $k$, and $\lim \left\{w_{k}\right\}_{k}=h_{b}$.

ProOF. $\left(b^{t}\right)^{\prime}=b^{t} \cdot \ln (b)$ which is $=\ln \left(g_{b}\right)<1$ at $t=g_{b}$ and $=\ln \left(h_{b}\right)>1$ at $t=h_{b}$. Let $s<1$. Apply Lemma 8 to $b^{t}$ on $\left(0, g_{b}\right]$ (regarded as $\left.g_{b}-t \mapsto g_{b}-b^{t}\right)$ to obtain $q_{1}$ and apply Lemma 8 to $b^{t}$ on $\left(g_{b}, h_{b}\right]$ (regarded as $\left.h_{b}-t \mapsto h_{b}-b^{t}\right)$ to obtain $q_{2}$; let $q=\min \left(q_{1}, q_{2}\right)$. Take the minimal $l$ satisfying $\exp _{b}^{l}(1)+1>g_{b}-q \cdot(1-s)^{2}$. Then for any $n$ we have

$$
(1-s)\left(\ln \left(g_{b}\right)\right)^{n}<\frac{g_{b}-\exp _{b}^{l+n}(1)}{g_{b}-\exp _{b}^{l}(1)}<(1+s)\left(\ln \left(g_{b}\right)\right)^{n}
$$

and since $h_{b}=g_{b}+1$ for any $m, n$ satisfying $\exp _{b}^{m}\left(\exp _{b}^{l+n}(1)+1\right)>h_{b}-q$ we have

$$
(1-s)\left(\ln \left(h_{b}\right)\right)^{m}<\frac{h_{b}-\exp _{b}^{m}\left(\exp _{b}^{l+n}(1)+1\right)}{g_{b}-\exp _{b}^{l+n}(1)}<(1+s)\left(\ln \left(h_{b}\right)\right)^{m}
$$

bringing together these inequalities we obtain

$$
\begin{aligned}
(1-s)^{2}\left(\ln \left(h_{b}\right)\right)^{m}\left(\ln \left(g_{b}\right)\right)^{n} & <\frac{h_{b}-\exp _{b}^{m}\left(\exp _{b}^{l+n}(1)+1\right)}{g_{b}-\exp _{b}^{l}(1)} \\
& <(1+s)^{2}\left(\ln \left(h_{b}\right)\right)^{m}\left(\ln \left(g_{b}\right)\right)^{n}
\end{aligned}
$$

Now, by the irrationality of $\ln \left(\ln \left(g_{b}\right)\right) / \ln \left(\ln \left(h_{b}\right)\right)$ one can choose $m, n$ so as to make $\left(\ln \left(h_{b}\right)\right)^{m}\left(\ln \left(g_{b}\right)\right)^{n}$ arbitrarily close to any positive real needed, in particular one can choose $\left(m_{k}, n_{k}\right)$ 's to approximate well from above each real of the form $(1-s)^{3 k} /(1+s)^{3 k}, k \in \mathbf{N}$. The corresponding $w_{k}=\exp _{b}^{m_{k}}\left(\exp _{b}^{l+n_{k}}(1)+1\right)$ 's satisfy the requirements of the claim: $(1+s)^{1-\varepsilon} /(1-s)^{1-\varepsilon}<\tilde{w}_{k} / \tilde{w}_{k+1}<(1+s)^{5} /(1-s)^{5}$, where $\tilde{w}_{i}=h_{b}-w_{i}$. (Divide the inequalities for $w_{i}$ 's.)

Claim 10. There exists $q>0$ such that $W$ is dense in $\left(h_{b}-q, h_{b}\right)$.

PROOF. For $s=1 / 2$ apply Lemma 8 to $b^{t}$ on $\left(0, g_{b}\right]$ to obtain $q_{1}, q_{1}<1$, and apply Lemma 8 to $b^{t}$ on $\left(g_{b}, h_{b}\right]$ to obtain $q_{2}, q_{2}<1$ (as in the beginning of Claim 9). Put $q=\frac{1}{2} \min \left(q_{1}, q_{2}\right)$. I will show that for each $u>0$ there exists an increasing sequence $\left\{v_{k}\right\}_{k} \subset W$ such that $v_{1}<h_{b}-q$ and $\&_{k} v_{k+1}-v_{k}<u$ with $\lim \left\{v_{k}\right\}_{k}=h_{b}$.

Apply Claim 9 with such an $s$ that $\left(w_{k+1}-w_{k}\right) /\left(h_{b}-w_{k}\right)<u / 6 q$ for each $k$. Let $n$ be the minimal positive integer such that $\exp _{b}^{n}\left(w_{1}\right)<h_{b}-q$. Then $\left\{v_{k}\right\}_{k}$, $v_{k}=\exp _{b}^{n}\left(w_{k}\right)$, satisfy the above requirements.

Since $\left(g_{b}, g_{b}+1\right)=\left(g_{b}, h_{b}\right)=\bigcup_{m=1}^{\infty} \exp _{b}^{m}\left(h_{b}-q, h_{b}\right)$ for any $q \in(0,1)$ part (iii) of the proposition is completed.

\section{REFERENCES}

1. R. Gurevic, Exponentiation of reals: effects of base choice, Abstracts Amer. Math. Soc. 4 (1983), 507, \#83T-99-387.

2. K. Kuratowski and A. Mostowski, Set theory, North-Holland, Amsterdam, 1967.

3. H. Levitz, The Cartesian product of sets and the Hessenberg natural product of ordinals, Czechoslovak Math. J. 29 (1974), 353-357.

Department of Mathematics, UNiversity of Illinois at URBana-Champaign, URBANA, ILLINOIS 61801 